\title{
Timely publication of all trial results may mean less overtreatment
}

\author{
Fiona Godlee editor, BMJ
}

Two BMJ campaigns coincide this week: for publication of all results from clinical trials, and against overdiagnosis and overtreatment.

Sentinel node biopsy (removal of the sentinel lymph node) is widely used to stage disease in patients with breast cancer and malignant melanoma. It carries a risk of lymphoedema and other complications, which increases if patients with positive sentinel nodes go on to have further lymph nodes removed in an effort to improve their survival. Evidence from several large trials has saved women with breast cancer from having axillary lymph node dissection if they have no clinical signs of axillary involvement: the trials found no significant difference in overall or disease free survival when axillary dissection was added to sentinel node biopsy.

But as Ingrid Torjesen reports, patients in the United Kingdom, the United States, and elsewhere who have malignant melanoma are routinely undergoing sentinel node biopsy, followed by regional lymphadenectomy if the biopsy is positive (doi:10. 1136/bmj.e8645). This is despite guidance from the UK's National Institute for Health and Clinical Excellence that there is, as yet, no published evidence from randomised controlled trials that sentinel node biopsy improves survival.

What trial evidence there is comes from the Multicenter Selective Lymphadenectomy trial (MSLT-1). Five year follow-up data published in the New England Journal of Medicine in 2006 found no overall survival advantage in patients who had sentinel node biopsy compared with those who didn't. But controversy has arisen over the authors' claims of improved disease free survival. These claims are based on a lower rate of nodal recurrence in the biopsy group, but critics say this finding is unsurprising, since the patients in the intervention group who are most at risk - those with a positive sentinel node — have had their regional nodes removed.

The researchers promised further analyses after 10 years of follow-up and at an interim point, and had these been published as expected in 2008 and 2011, the controversy might have been resolved by now. But these data have not yet been published. The authors say they are working on their analysis. Meanwhile, sentinel node biopsy has become the standard of care in many countries. NICE says the procedure is justified only in the context of clinical trials, but Torjesen has learnt that it is carried out in at least 19 trusts in England, only two of which are involved in ongoing trials. Is this another example of harmful overtreatment? Only the trial data will tell.

This is not the only case in which clinical trial data have been published late or not at all. In addition to the well documented suppression of data by drug companies, there are clear examples of delayed or non-publication in non-industry sponsored trials. Last week we reported on the long delay in publication of a trial of deworming and vitamin A that was completed in 2005 (BMJ 2013;346:e8558). This week in an editorial we reference a large trial of adenoidectomy that has only just reported after nearly a decade (doi:10.1136/bmj.f105). The editorial calls for ethics committees, funders, and institutions to take responsibility for ensuring that the trials they approve, support, and host are published in full and in good time. Working with others, we are taking the campaign to patients and the public. If you agree that all clinical trials should be published, sign the petition at alltrials. net.

Cite this as: BMJ 2013;346:f159

๑ BMJ Publishing Group Ltd 2013 